\title{
Weekly Cyclophosphamide-Bortezomib- Dexamethasone Induction Performs Comparably to Twice-Weekly Dosing with Respect to Both Response Rates and Survival after Autologous Transplant
}

\author{
Caitlin Thirunavukarasu $^{\mathrm{a}}$ Nicholas Weber ${ }^{\mathrm{a}}$ Kirk Morris $^{\mathrm{a}}$ Elango Subramonia Pillai ${ }^{\mathrm{a}}$ \\ Cameron Curley $^{\mathrm{a}}$ Jason Butler ${ }^{\mathrm{a}}$ Glen Kennedy ${ }^{\mathrm{a}, \mathrm{b}}$ \\ a Department of Haematology, Cancer Care Services, Royal Brisbane and Women's Hospital, Brisbane, QLD,

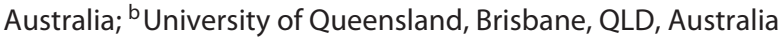

Dear Editor,

We read with interest the study by Tanaka et al. [1] prospectively evaluating the efficacy and safety of weekly cyclophosphamide-bortezomib-dexamethasone (CBD) induction in transplant-eligible patients with newly diagnosed multiple myeloma. Response rates by intention-totreat were lower than previously reported, and whilst this may have been attributable to cyclophosphamide dose, the authors conclude that weekly CBD may be less effective than twice-weekly approaches.

In Australia, CBD is the most commonly used induction regimen for transplanteligible patients. Weekly bortezomib dosing is preferred based on the more favourable toxicity profile, although no randomised comparisons with the original twice-weekly regimens have been conducted in this patient group. Furthermore, the phase II studies of weekly CBD employed a bortezomib dose of $1.5 \mathrm{mg} / \mathrm{m}^{2}$, which is not reimbursed in Australia [2,3].

We sought to compare outcomes for twice-weekly and once-weekly CBD regimens in patients who underwent autologous stem cell transplant (ASCT) in our centre since the approval of bortezomib for front-line therapy in 2012. Data was col- lected on patients with newly diagnosed multiple myeloma as defined by International Myeloma Working Group (IMWG) criteria [4], who were treated with bortezomib induction and underwent ASCT as part of primary therapy. Patients who underwent ASCT for relapsed disease or with incomplete bortezomib dosing details were excluded.

Patients received physician-directed twice-weekly or weekly CBD for 4 cycles (maximum 16 bortezomib doses) with either subcutaneous or intravenous (IV) bortezomib at $1.3 \mathrm{mg} / \mathrm{m}^{2}$ on days $1,4,8$ and 11 (twice-weekly cohort, 21-day cycle) or days 1, 8, 15 and 22 (once-weekly cohort, 28-day cycle). Oral cyclophosphamide $300 \mathrm{mg} / \mathrm{m}^{2}$ was administered weekly. Dexamethasone $20 \mathrm{mg}$ was administered on days $1,2,4,5,8$, 9, 11 and 12 (twice-weekly cohort) or $40 \mathrm{mg}$ on days 1, 8, 15 and 22 (weekly cohort). All patients underwent peripheral blood stem cell mobilisation using cyclophosphamide 2 $\mathrm{g} / \mathrm{m}^{2}$ and filgrastim $10 \mu \mathrm{g} / \mathrm{kg}$ and proceeded to ASCT with melphalan conditioning (200 $\mathrm{mg} / \mathrm{m}^{2} \mathrm{IV}$ on day -1 , dose reduced for age or renal impairment) with tandem ASCT and/or post-transplant maintenance therapy performed at physician discretion.
Response endpoints included IMWGdefined overall response rate (ORR) and rate of very good partial response (VGPR) or better after induction and at day 100 after ASCT compared between groups using the $\chi^{2}$ test. Survival endpoints were calculated using Kaplan-Meier analysis.

Sixty patients treated between October 2012 and June 2017 were included in the analysis. 31 patients (52\%) received twiceweekly bortezomib and 29 patients (48\%) received weekly bortezomib. There were no significant differences between groups with respect to age, high-risk cytogenetics, ISS stage, cumulative bortezomib dose, route of bortezomib administration (IV vs. subcutaneous) or use of post-ASCT maintenance therapy. Post-induction ORR for the twice-weekly and once-weekly groups were 87 and $86 \%$, respectively (OR 0.93 , $95 \%$ CI $0.21-0.41, p=0.92$ ), while VGPR or better was achieved in 61 and $45 \%$, respectively (OR 0.51, 95\% CI 0.18-1.44, $p=$ $0.20)$. Four patients in each group achieved less than a partial response to $\mathrm{CBD}$, of whom 5 received salvage therapy; all underwent ASCT and were included in the final analysis. VGPR or better at day 100 after ASCT was achieved in 24/31 (77\%) in karger@karger.com

(C) 2019 S. Karger AG, Basel

www.karger.com/aha

Karger ${ }^{\prime \prime}=$
Nicholas Webe

Department of Haematology, Cancer Care Services

Royal Brisbane and Women's Hospital

Butterfield Street, Herston, Brisbane, QLD 4006 (Australia)

E-Mail nicholas.weber@ health.qld.gov.au 


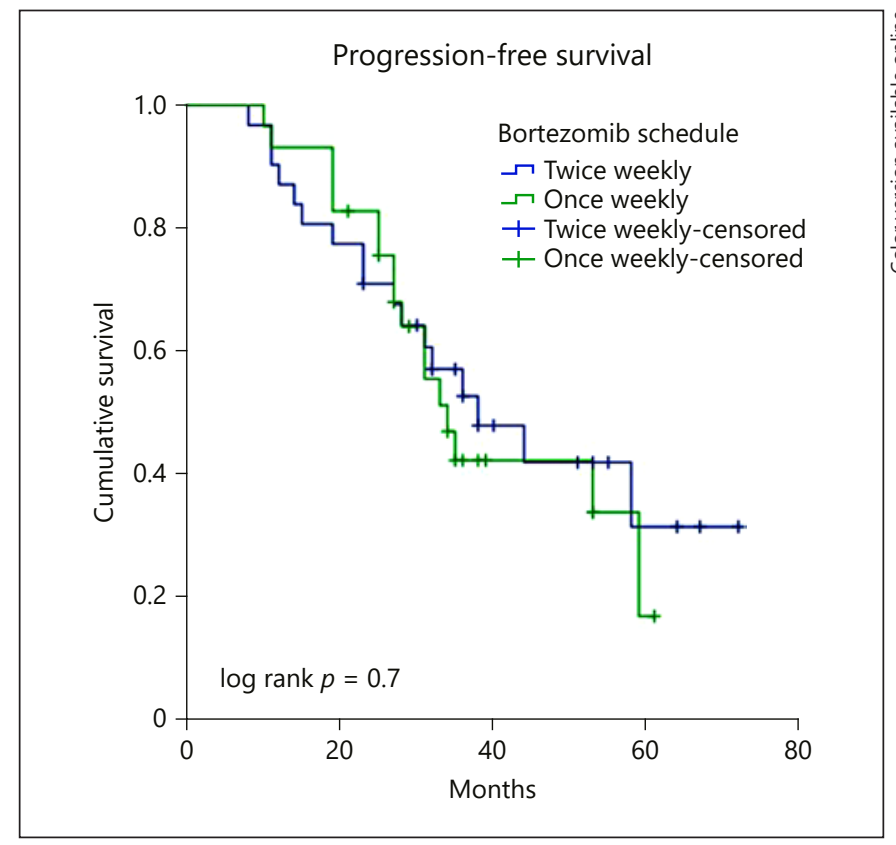

Fig. 1. Progression-free survival.

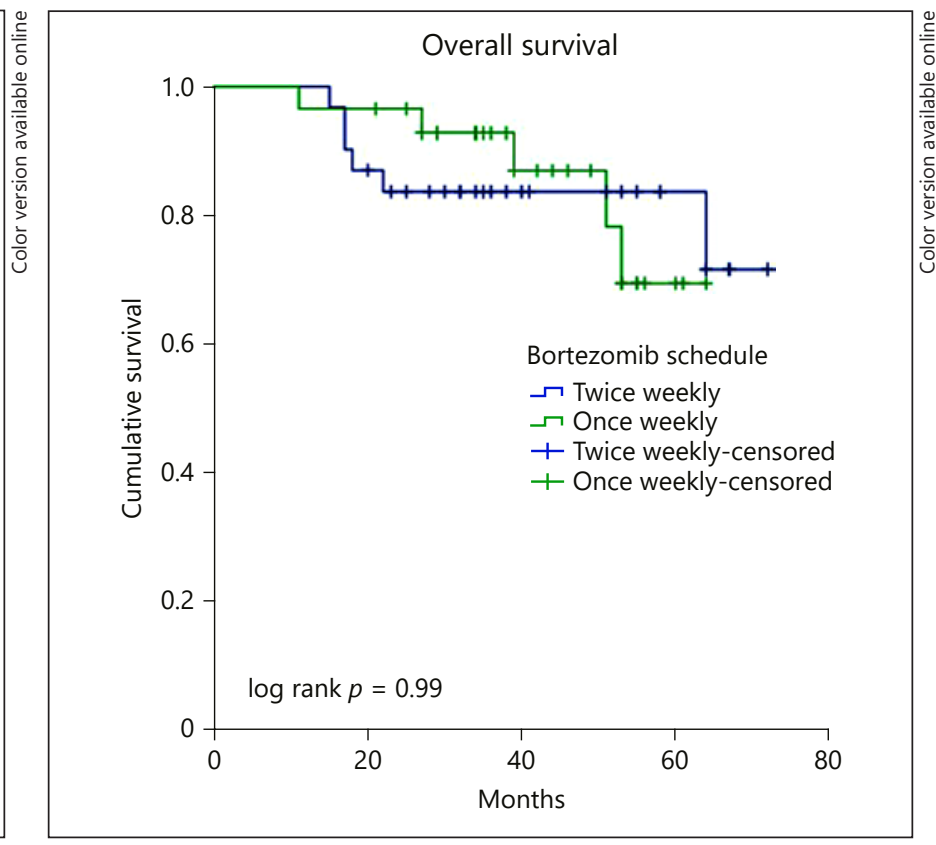

Fig. 2. Overall survival. the twice-weekly group and $21 / 29(72 \%)$ in the once-weekly group (OR $0.77,95 \% \mathrm{CI}$ $0.24-2.47, p=0.66$ ).

Median follow-up duration for the entire cohort was 42 months. Median progression-free survival was 38 months in the twice-weekly group and 34 months in the weekly group (HR 1.14, 95\% CI 0.58-2.24, $p=0.71$ ) (Fig. 1), while median overall survival has not been reached in either group. The 3-year overall survival was 84 and $93 \%$, respectively ( $p=0.99)$ (Fig. 2 ).

We conclude that, within the limitations of our retrospective study design, weekly CBD induction performs comparably to twice-weekly dosing with respect to both response rates and survival. As reported by Tanaka et al. [1], we see a trend towards lower VGPR or better rate with weekly CBD, but this does not appear to affect outcomes after ASCT. This is consistent with other published "real-world" studies employing weekly bortezomib at the $1.3 \mathrm{mg} / \mathrm{m}^{2}$ dose [5]. We support the use of weekly bortezomib dosing given comparable outcomes while minimising widely reported toxicity associated with twiceweekly regimens.

\section{References}

1 Tanaka K, Toyota S, Akiyama M, Wakimoto N, Nakamura Y, Najima Y, et al.; on behalf of the Ochanomizu Hematology Study Group. Efficacy and Safety of a Weekly Cyclophosphamide-Bortezomib-Dexamethasone Regimen as Induction Therapy Prior to Autologous Stem Cell Transplantation in Japanese Patients with Newly Diagnosed Multiple Myeloma: A Phase 2 Multicenter Trial. Acta Haematol. 2019;141(2):111-8.
2 Reeder CB, Reece DE, Kukreti V, Chen C, Trudel S, Hentz J, et al. Cyclophosphamide, bortezomib and dexamethasone induction for newly diagnosed multiple myeloma: high response rates in a phase II clinical trial. Leukemia. 2009 Jul;23(7):1337-41.

3 Reeder CB, Reece DE, Kukreti V, Chen C, Trudel S, Laumann K, et al. Once- versus twice-weekly bortezomib induction therapy with CyBorD in newly diagnosed multiple myeloma. Blood. 2010 Apr;115(16): 3416-7.

\section{Disclosure Statement}

The authors have no conflicts of interest to declare.

\section{Funding Sources}

No funding was received for this study.

\section{Author Contributions}

C.T., N.W., K.M., E.S.P., C.C., J.B. and G.K. acquired data. C.T. and N.W. wrote the manuscript. All authors approved the final paper.

4 Kumar S, Paiva B, Anderson KC, Durie B, Landgren $\mathrm{O}$, Moreau $\mathrm{P}$, et al. International Myeloma Working Group consensus criteria for response and minimal residual disease assessment in multiple myeloma. Lancet Oncol. 2016 Aug;17(8):e328-46.

5 Ong SY, Ng HY, Surendran S, Linn YC, Chen Y, Goh YT, et al. Subcutaneous bortezomib combined with weekly cyclophosphamide and dexamethasone is an efficient and well tolerated regime in newly diagnosed multiple myeloma. Br J Haematol. 2015 Jun;169(5):754-6. 